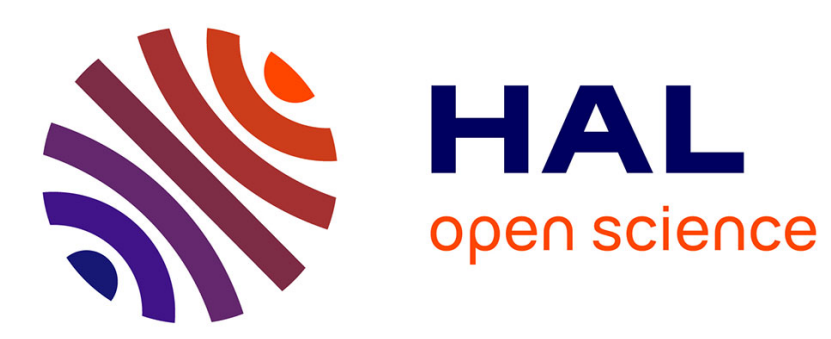

\title{
Comment une chimère est devenue une politique internationale : l'arène de la " paix positive "
}

Sandrine Lefranc

\section{To cite this version:}

Sandrine Lefranc. Comment une chimère est devenue une politique internationale : l'arène de la " paix positive ". Critique Internationale, 2021, № 92 (3), pp.95-120. 10.3917/crii.092.0098 . hal03503168

\section{HAL Id: hal-03503168 \\ https://cnrs.hal.science/hal-03503168}

Submitted on 2 Jan 2022

HAL is a multi-disciplinary open access archive for the deposit and dissemination of scientific research documents, whether they are published or not. The documents may come from teaching and research institutions in France or abroad, or from public or private research centers.
L'archive ouverte pluridisciplinaire HAL, est destinée au dépôt et à la diffusion de documents scientifiques de niveau recherche, publiés ou non, émanant des établissements d'enseignement et de recherche français ou étrangers, des laboratoires publics ou privés. 


\title{
Comment une chimère est devenue une politique internationale L'arène de la « paix positive »
}

\author{
Sandrine Lefranc
}

Sandrine Lefranc est politiste au CNRS (CEE, Sciences Po Paris), où elle mène des recherches sur différentes formes de justice - transitionnelle, pénale, restaurative - ainsi que sur les politiques de paix et de mémoire. Parmi ses publications récentes : avec Sarah Gensburger, Beyond Memory. Can We Really Learn From the Past?, Palgrave Macmillan Memory Studies, 2020 ; avec Delphine Griveaud, «La justice transitionnelle, un monde-carrefour. Contribution à une sociologie des professions internationales », Cultures \& Conflits, 119-120, 2020, pp. 3965 , et «Des «procès rwandais » à Paris. Échos locaux d'une justice globale », Droit et société, 2(102), 2019, pp. 299-318. Courriel : sandrine.lefranc@cnrs.fr

\section{Résumé}

En contrepoint des modalités usuelles d'intervention des organisations internationales en faveur de la paix - militaires, humanitaires, par le développement économique et la réforme des institutions -, une arène s'est développée autour des ambitions de consolidation de la paix (peacebuilding) et de "paix positive». Les initiatives prises dans ce cadre contournent les élites des gouvernements, pour susciter le dialogue et construire, à l'échelle locale, des liens entre les gens "ordinaires». Confrontées à ce que les sciences sociales disent de la guerre et de la paix, ces pratiques semblent chimériques. Comment expliquer alors leur diffusion depuis la fin de la Guerre froide? Cet article montre, à rebours des hypothèses privilégiées par l'étude des relations internationales et à partir de l'étude d'une diversité d'organisations non gouvernementales appréhendées comme des maillons d'une chaîne d'intervention, que l'arène de la transformation des conflits n'est pas née d'un projet impérialiste ou d'un contre-projet militant. La proposition a priori nä̈ve d'une paix positive tire sa force et sa capacité de structurer des interventions internationales, des causes locales (principalement aux ÉtatsUnis) qu'elle transpose à l'échelle internationale, d'une part, et, d'autre part, de la constitution "en miroir », autour d'une proposition critique, de l'espace académique et des politiques internationales de paix. Ce sont ces projections, ces rencontres et ces interdépendances qui, d'une chimère, font une politique.

Les organisations internationales sont susceptibles d'intervenir militairement dans les pays qui sortent d'un conflit civil violent. Elles leur destinent une aide humanitaire, appuient leur développement économique, réforment leurs institutions. Mais elles font davantage, et autrement: leurs agents travaillent à la consolidation de la paix (peacebuilding) et à l'installation d'une «paix positive». Les initiatives nombreuses qu'ils prennent alors contournent les élites et les gouvernements, pour susciter le dialogue et construire, à l'échelle locale, des liens entre les gens « ordinaires »- selon une expression courante. Confrontées à ce que les sciences sociales disent des processus de guerre et de paix, ces pratiques semblent chimériques. Comment expliquer alors leur diffusion depuis la fin de la Guerre froide ?

Le terme de peacebuilding est devenu depuis 1992 un maître-mot de l'action de l'Organisation des Nations Unies (ONU) en matière de paix. Aux modalités usuelles de négociation d'accords de paix et d'intervention militaire (preventive diplomacy, peacemaking et peacekeeping), il ajoute «l'action menée en vue de définir et d'étayer les structures propres à raffermir la paix afin d'éviter une reprise des hostilités ${ }^{1}$. Le rapport Brahimi a creusé ce

\footnotetext{
${ }^{1}$ United Nations, An Agenda for Peace. Preventive Diplomacy, Peacemaking and Peace-Keeping. Report of the SecretaryGeneral, A/47/277, 17 juin 1992 ; United Nations, Supplement to an Agenda for Peace: Position Paper of the Secretary-
} 
sillon en revendiquant, en 2000, l'objectif de la construction de «something that is more than just the absence of war $»^{2}$; cinq ans plus tard, une Peacebuilding Commission a été créée. Des salariés d'organisations internationales (OI), des universitaires, des missionnaires et des fonctionnaires d'agences nationales de coopération, ont plus largement donné à cette finalité de la consolidation de la paix une application particulière, qui renoue avec l'ambition d'une autre expression, académique celle-ci : la «paix positive », que Johan Galtung a opposé à cette paix «négative » qu'est la cessation de la violence physique négociée par les élites politiques ${ }^{3}$. L'idée est d'en finir durablement avec la violence y compris structurelle, en créant les conditions d'une coopération fondée sur l'égalité, la justice sociale, l'autonomie des citoyens et d'autres caractéristiques prêtées à une « paix juste ».

Que font les promoteurs internationaux d'une telle paix ? Ils organisent, aux côtés d'acteurs locaux, des dialogues au cours desquels une dizaine de personnes - appartenant aux élites intermédiaires ou simples quidams - évoquent ensemble le conflit. Ils financent des festivals artistiques et des événements sportifs célébrant les vertus de la paix, ou produisent des feuilletons télévisuels racontant la difficile coexistence d'ethnies différentes au sein d'un quartier. D'autres promeuvent des échanges entre victimes du conflit violent et criminels politiques, qui doivent permettre la diffusion d'un récit historique - dans le cadre par exemple des commissions de vérité. Ils appliquent ce faisant des méthodes formalisées par des universitaires et des experts. Il s'agit, pour ces pacificateurs venus de pays étrangers, d'œuvrer à la création de liens denses entre les individus, en même temps que de leur faire construire un monde commun. Ils envisagent symétriquement la violence politique comme la complète destruction des institutions, du lien social et des psychologies individuelles. Ces organisations s'échinent ainsi à réconcilier les sociétés affectées par un conflit : recoudre un tissu social déchiré par la guerre, faire dialoguer les groupes ennemis, apaiser les victimes, favoriser le dépassement par tous des préjugés et le développement d'interactions sociales apaisées.

Or, leurs agents, formés aux sciences sociales, souvent même impliqués dans la recherche et l'enseignement, se font ainsi les avocats d'une conception de la paix et de la violence dont d'autres discussions scientifiques montrent la faiblesse. L'école réaliste défend en effet, au sein de la discipline des relations internationales, une conception négative de la paix, tandis que les anthropologues mettent en évidence les dimensions positives de la violence - au sens de sa capacité de créer du lien et de la solidarité - et que les sociologues analysent la faiblesse de la paix positive dans les sociétés stables. Cette paix nourrie par les récits communs, la reconnaissance de la souffrance et les interactions entre ex-ennemis, d'ailleurs, ses promoteurs ne jureraient pas qu'elle soit réalisée dans leurs pays d'origine. Qui prétendrait en effet qu'aux États-Unis (lieu d'origine ou de formation prédominant des agents ${ }^{4}$ ) des liens étroits existent entre les groupes raciaux et sociaux ? Qui affirmerait que les sociétés européennes sont des lieux où la mixité sociale et culturelle est forte, et où tous les habitants partagent une même lecture des conflits passés? Ces femmes et hommes travaillant pour des organisations internationales, souvent critiques des conditions sociales dans leurs pays d'appartenance, n'ignorent pas que la paix qu'ils entreprennent d'installer dans des pays hier parfois théâtres de massacres, n'est pas un état social si courant.

Lorsqu'on les interroge, ils reconnaissent aisément que leur action de transformation des conflits (terme qu'ils préfèrent à celui de gestion ou résolution des conflits) est, au mieux, un pari. Ils savent que beaucoup de leurs interventions n'ont pas eu le succès escompté. Ils n'ignorent pas non plus que leur action est fragilisée par le manque de coordination entre les acteurs de la reconstruction des pays dits post-conflit, ou par les détournements dont leurs programmes peuvent faire l'objet. Et pourtant ils continuent à agir, partout, dans le sens de cette paix voulue juste et positive. Ils l'ont fait d'ailleurs avec un succès croissant à partir de la

General on the Occasion of the Fiftieth Anniversary of the United Nations, janvier 1995 https://digitallibrary.un.org/record/168325. Consultés le 13 août 2020.

${ }^{2}$ United Nations, Report of the Panel on United Nations Peace Operations, A/55/305-S/2000/809, 21 août 2000, p. 3.

3 Johan Galtung, «Violence, Peace, and Peace Research », Journal of Peace Research, 6(3), 1969, pp. 167-191s.

${ }^{4}$ Et de création de trois quart des ONG spécialisées (dans le cas de l'Alliance for Peacebuilding, voir infra). 
fin de la Guerre froide. Les budgets des gouvernements et des OI alloués au peacebuilding ${ }^{5}$ ont augmenté, les liens se sont étoffés entre OI, États et organisations non gouvernementales (ONG) généralistes ou spécialisées dans les actions de transformation des conflits. Le contexte de la « guerre contre le terrorisme » a ralenti ce déploiement, mais n’y a pas mis fin ${ }^{6}$.

Comment expliquer que des modalités d'action qui peuvent apparaître naïves, des conceptions de la paix peut-être trop ambitieuses, soient devenues une composante à part entière de l'intervention internationale? La littérature de relations internationales propose des explications. Les théories constructivistes mettent l'accent sur l'entêtement de communautés d'idées, qui peuvent un jour l'emporter après avoir longtemps paru naïves ${ }^{7}$. Les auteurs réalistes pointent l'instrumentalisation de ces chimères par des États puissants légitimant ainsi une intervention intéressée dans un pays post-conflit, tandis que leurs pairs plus critiques voient dans la plupart des formes du peacebuilding un néo-impérialisme hypocrite ${ }^{8}$; la paix positive n'est plus alors qu'une critique interne au libéralisme, située au centre-gauche ${ }^{9}$. D'autres encore voient dans ces formes d'intervention l'autolégitimation de communautés d'expatriés enfermées sur elles-mêmes ; «"Peacebuilding" has become a lifestyle for a small community of global "cosmopolitans" $\gg 10$.

Cet article veut montrer que la diffusion internationale de cette chimère n'est imputable ni à l'idiotie des acteurs ${ }^{11}$, ni à une mauvaise intention néo-libérale. Elle est le produit de la constitution d'une arène internationale autour d'une proposition dont la nature est d'abord critique. Cette proposition, centrée sur la transformation du conflit et la construction d'une paix positive, a été faite en effet en réaction au paradigme et aux formes d'intervention dominants de la paix "négative », c'est-à-dire une conception de la guerre et de la paix réaliste, institutionnelle et élitaire. La paix positive est de ce fait critique aussi de la centralité de l'État dans le gouvernement international de la violence.

La diffusion internationale d'une définition empiriquement peu fondée de la paix est intéressante en effet parce qu'elle met sur la piste des manières dont un milieu international hétérogène s'accorde sur une définition de la paix et les moyens de l'atteindre. Le terme d'arène, distingué d'un espace libre de discussion entre pairs aussi bien que d'un champ autonome et structuré ${ }^{12}$, est utile de deux manières. En premier lieu, le terme invite à l'analyse

\footnotetext{
${ }^{5}$ Le peacebuilding, au sens large privilégié notamment par l'ONU, comporte trois dimensions : la sécurité, la réforme des institutions de l'État et ce que beaucoup appellent les «dimensions socioéconomiques du conflit », qui incluent des termes aussi divers que la justice transitionnelle et la réconciliation, le «trauma counseling » et les dialogues en vue de «building bridges between different communities ", Michael Barnett, Hunjoon Kim, Madalene O'Donnell et Laura Sitea, «Peacebuilding: What is in a Name? », Global Governance, 13, 1, 2007, pp. 48-50. Le concept de paix positive, et celui lié de transformation des conflits, sont les fils directeurs communs à ces termes et activités.

${ }^{6}$ Les dépenses des États membres de l'OCDE pour la catégorie « conflit, paix et sécurité » sont passées de 1 milliard de dollars E.-U. en 2004 à 3,2 milliards en 2008 puis à 5,4 en 2018 (arrondis au dixième) : https://stats.oecd.org/viewhtml.aspx?datasetcode=TABLE5\&lang=en\#. Consulté le 11 novembre 2020.

${ }^{7}$ Margaret E. Keck et Kathryn Sikkink, Activists Beyond Borders: Advocacy Networks in International Politics, Ithaca, Cornell University Press, 1998 ; Martha Finnemore, The Purpose of Interventions. Changing Beliefs about the Use of Force, Ithaca, Cornell University Press, 2003.

${ }^{8}$ Edward Newman, «Liberal Peacebuilding Debates », in E. Newman, R. Paris et O. Richmond, ed., New Perspectives on Liberal Peacebuilding, New York: United Nations University Press, 2009, pp. 26-53.

${ }^{9}$ Selon D. Sharp, le concept « strongly resonates with some of the ideals of left-of-center modern-day progressives around the globe », « Positive Peace, Paradox, and Contested Liberalisms », International Studies Review, 22 (1), 2020, p. 128.

${ }_{10}$ Tobias Denskus, «Peacebuilding Does Not Build Peace», Development in Practice, 17, 4-5, août 2007, p. 660. Voir Séverine Autesserre, Peaceland: Conflict Resolution and the Everyday Politics of International Intervention, Cambridge, Cambridge University Press, 2014, et Catherine Goetze, The Distinction of Peace: A Social Analysis of Peacebuilding, Ann Arbor, University of Michigan Press, 2017.

${ }^{11}$ En référence à l'idiot de Garfinkel dépeint par Ruwen Ogien, qui explique que les gens se saluent par des attentes fondées sur des valeurs de courtoisie, face à un idiot symétrique, utilitariste, lui. «L'idiot de Garfinkel», in M. de Fornel et alii, L'ethnométhodologie, Paris, La Découverte, 2001, pp. 57-74.

${ }^{12} \mathrm{C}$ 'est-à-dire un secteur de la société relativement autonome régi par des règles et poursuivant des fins qui lui sont propres, et impliquant une coupure entre professionnels et profanes, Pierre Bourdieu, Les règles de l'art. Genèse et structure du champ littéraire, Paris, Le Seuil, 1992. Pour une tentative d'application aux mobilisations internationales, voir Yves Dezalay et Bryant G. Garth, La mondialisation des guerres de palais. La restructuration du pouvoir d'État en Amérique latine, entre notables du droit et «Chicago Boys », Paris, Le Seuil, 2002, et l'article de l'auteure avec Delphine Griveaud, "La justice transitionnelle, un monde-carrefour. Contribution à une sociologie des professions internationales », Cultures \& conflits, 119120, 2020, pp. 39-65.
} 
du fonctionnement collectif d'une diversité d'organisations, de statut et d'importance variés, qui ont en commun d'embrasser ces positions critiques ${ }^{13}$. L'arène de la transformation des conflits se caractérise, en deuxième lieu, par des situations d'interaction, de collaboration et de concurrence, entre des organisations qui mobilisent les ressources de l'international ${ }^{14}$, mais articulent une variété de mondes sociaux, à l'échelle internationale aussi bien que locale. Elle englobe en effet les États, les OI, mais aussi des acteurs pris dans des activités domestiques qui ont a priori peu à voir avec les relations internationales. La guerre, la paix, les outils utilisés, sont par conséquent définis «en miroir» des enjeux et des routines professionnelles des acteurs dans leurs pays d'origine, et au fur et à mesure de leurs rencontres dans l'arène internationale (au siège des organisations, sur les terrains d'intervention, lors des colloques et formations). Si l'idée de la paix positive se diffuse, c'est ainsi non du fait de sa fonctionnalité, de la conviction ou de la position dominante de leurs promoteurs, mais parce que cette arène fonctionne comme un carrefour entre des mondes locaux différenciés, souvent distants des gouvernements... C'est ainsi que des chimères en viennent à être défendues, et même mises en œuvre, sur la scène internationale.

\section{Transformer les conflits violents, l'autre voie du gouvernement international de la violence?}

\section{La paix positive, une proposition critique}

La paix positive est une proposition critique - une $«$ critical peacebuilding theory ${ }^{15}-$ qui se distingue des modalités prédominantes du gouvernement international de la violence intraétatique, et demeure secondaire vis-à-vis d'elles ${ }^{16}$. Elle s'oppose bien sûr à l'usage de la force à l'encontre d'États jugés fauteurs de guerres ; le contexte immédiatement contemporain de «guerre contre le terrorisme» a d'ailleurs atténué sa visibilité. Elle se distingue aussi de l'interposition militaire dans les conflits armés ${ }^{17}$. Mais elle n'est pas seulement une proposition d'action non-violente. Elle prend aussi le contrepied des stratégies d'action ciblant les seules élites politiques et institutions d'État. La paix que ses promoteurs remettent en question et entendent compléter est négative, en ce sens qu'elle est un accord passé entre des responsables politiques et militaires, qui le déferaient volontiers si la violence était susceptible de mieux les servir. Alors que ces négociations débouchent sur des accords fragiles, gagés sur le seul intérêt du moment des signataires, la paix positive entend intéresser et convaincre durablement l'ensemble d'une société, créer des relations de confiance entre tous ses membres au travers de rencontres informelles opposées aux rituels diplomatiques ${ }^{18}$.

Cette proposition n'est donc pas formulée, principalement, par ce canal principal, étatique, des politiques de paix que sont les diplomates. En lieu et place des États et des OI, ce sont souvent des acteurs "privés » (figures éminentes, hommes d'Églises, universitaires, ONG, villes) qui intercèdent auprès d'élites intermédiaires ${ }^{19}$. La paix positive s'est par exemple incarnée à partir des années 1960 dans des exercices de médiation, des conférences réunissant

\footnotetext{
${ }^{13}$ Nancy Fraser, critiquant la notion d'espace public, insiste sur l'existence d' « arènes discursives parallèles dans lesquelles les membres des groupes sociaux subordonnés élaborent et diffusent des contre-discours », Qu'est-ce que la justice sociale ? Reconnaissance et redistribution, Paris, La Découverte, 2005, p. 126.

${ }^{14}$ Michel Dobry, Sociologie des crises politiques. La dynamique des mobilisations multisectorielles, Paris, Presses de Sciences Po, 2009, rééd., p. 117.

${ }_{15}$ Simon Robins, «Mapping a Future for Transitional Justice by Learning From its Past», International Journal of Transitional Justice, 9(1), 2015, pp. 181-190.

${ }^{16}$ Ce caractère secondaire par rapport aux modalités « violentes » du gouvernement de la violence se traduit sur le plan des finances. Les ONG étudiées ici, et par exemple celles qui sont membres de l'Alliance for Peacebuilding, sont généralement de petite taille et disposent d'un budget de moins de $500000 \$$ annuels pour $60 \%$ d'entre elles (https://www.allianceforpeacebuilding.org).

${ }^{17}$ Rama Mani, Beyond Retribution. Seeking Justice in the Shadow of War, Cambridge, Polity/Blackwell, 2002, p. 4.

${ }^{18}$ Kumar Rupesinghe et Sanam N. Anderlini, Civil Wars, Civil Peace, An Introduction to Conflict Resolution, Londres, Pluto Press, 1998, p. 131 (les auteurs sont membres d'International Alert, ONG évoquée ici).

${ }^{19}$ John Paul Lederach, The Moral Imagination: The Art and Soul of Building Peace, Oxford, Oxford University Press, 2005.
} 
scientifiques et élites sociales ${ }^{20}$, ou des ateliers de «résolution des problèmes »: autant de variations d'un principe de dialogue ouvert à des individus éclairés mais sans responsabilité politique directe, susceptibles d'entrer dans des relations de coopération avec leurs ennemis présumés $^{21}$. Les conflits américano-soviétique, israélo-palestinien, chypriote ont été autant de contextes d'expérimentation $^{22}$. Depuis, et à partir surtout des années 1980, ce sont des ONG internationales spécialisées comme Search for Common Ground (SFCG), qui ont entrepris de systématiser ce principe de dialogue à l'ensemble des populations affectées - à l'échelle des villages, notamment, et à l'attention des élites locales aussi bien que des femmes et enfants « ordinaires ». Des espaces de rencontre mixtes ont été créés : dans les écoles, les médias, les quartiers, lors d'événements artistiques ou sportifs ou encore sur les places de marché ou dans le cadre d'activités économiques ${ }^{23}$.

Ces formes d'intervention se distinguent des modalités usuelles de pacification dans l'espace international, parce qu'elles sont conçues pour s'en distinguer. Non seulement ces activités sont portées par des acteurs relativement marginaux sur la scène des relations internationales, mais elles ont été esquissées surtout dans un autre monde : le monde universitaire, aux ÉtatsUnis et ailleurs. À partir des années 1950, c'est en effet l'université qui abrite le développement d'une science nouvelle : la peace research, qui entreprend de rassembler les connaissances sur les conflits et les processus de résolution des conflits dans tous les secteurs de la vie sociale. Ces scientifiques ne s'intéressent pas en effet qu'aux conflits internationaux ; certains d'entre eux ont travaillé d'abord sur les conflits ethno-raciaux mais aussi sur les conflits du travail ou même familiaux ${ }^{24}$, où ils explorent les différentes voies d'une résolution des litiges alternatives par rapport au droit et à l'État.

Lorsqu'ils se sont intéressés à l'international, ils se sont opposés frontalement aux conceptions dominantes du conflit dans les relations internationales. Au paradigme réaliste concédant un statut inévitable à la guerre dans un monde international anarchique régi par les intérêts des plus puissants des États, ils ont préféré les paradigmes concurrents que sont l'idéal wilsonien pacifiste d'éducation des élites politiques œuvrant à la sécurité collective, et les conceptions libérale et constructiviste des relations internationales ${ }^{25}$. Les internationalistes en retour ne s'y sont guère intéressés; la peace research n'était guère qu'un carrefour interdisciplinaire, associant des sociologues, des mathématiciens et des psychologues.

Mais la position critique de ces spécialistes de la paix ne s'explique pas simplement par leur marginalité institutionnelle en regard des disciplines universitaires établies. Ce sont, aussi, souvent des militants: pacifistes, militants des droits civiques, et membres de groupes religieux amenés à affirmer des positions critiques vis-à-vis des interventions militaires d'État. Évangéliques, Quakers, mennonites y sont nombreux ${ }^{26}$. À la critique politique des élites s'est

\footnotetext{
${ }^{20}$ Les conférences de Dartmouth sont par exemple organisées à partir de 1961 pour faire dialoguer des élites étatsunienne et soviétique, sur des questions d'apparence technique, dans un cadre universitaire, avec l'aval des gouvernements mais en leur absence. Elles passent pour avoir eu une réelle influence sur les responsables soviétiques à l'origine de la libéralisation, Matthew Evangelista, Unarmed Forces: the Transnational Movement to End the Cold War, Cornell University Press, 1999.

${ }^{21}$ Ce principe du dialogue en petit groupe a pris des formes variées : problem-solving workshop avec la médiation d'un universitaire étranger, ou dialogue régulé par un responsable d'ONG locale entre femmes ou jeunes issus des groupes ennemis, par exemple. Ronald J. Fisher, «Social-Psychological Processes in Interactive Conflict Analysis and Reconciliation », in M. Abu-Nimer, ed., Reconciliation, Justice, and Coexistence: Theory and Practice, Lanham, Lexington Books, 2001, p. 25.

${ }^{22}$ Voir Herbert C. Kelman, «The Political Psychology of the Israeli-Palestinian Conflict: How Can We Overcome the Barriers to a Negotiated Solution? », Political Psychology, 8 (3), 1987, pp. 347-363 ; John W. Burton, Conflict: Resolution and Provention, New York, Macmillan, 1990.

${ }^{23}$ De l'auteure, « Du droit à la paix. La circulation des techniques internationales de pacification par le bas », Actes de la recherche en sciences sociales, 174 , septembre 2008, pp. 48-67.

${ }^{24}$ Ibid.; Charles Tenenbaum, La médiation dans les relations internationales. Évolutions et transformations depuis 1945, Thèse de doctorat, Institut d'Études Politiques de Paris, 2010 ; Martha Harty et John Modell, «The First Conflict Resolution Movement, 1956-1971. An Attempt to Institutionnalize Applied Interdisciplinary Social Science », The Journal of Conflict Resolution, 35(4), décembre 1991, pp. 720-758.

${ }^{25}$ Lesquelles valorisent toutes deux la coopération internationale, favorisée par des acteurs non-étatiques ou la diffusion d'idées. Dario Battistella, Théories des relations internationales, Paris, Presses de Sciences Po, 2015, rééd.

${ }^{26}$ De l'auteure, «Des pacificateurs inspirés. Notes sur des groupes anabaptistes et évangéliques américains », Terrain, 51, septembre 2008, pp. 42-49.
} 
ainsi ajoutée celle du canal étatique lui-même par des organisations contestant le pouvoir séculier.

Cette critique scientifique conteste ainsi la spécificité des relations internationales comme la centralité des États. La conception même d'une paix positive suppose en effet une dévalorisation du rôle des élites ${ }^{27}$. Elle dénonce un ordre libéral, néo-impérial, masculiniste ${ }^{28}$. Elle s'oppose parfois frontalement à une «idéologie du management » ${ }^{29}$, c'est-à-dire à des formes de résolution des conflits qui insistent sur la recherche de l'accord, facilitée par un tiers neutre. Les « dialoguistes » se définissent par opposition aux «négociateurs ». Les seconds privilégient la négociation et considèrent le pouvoir politique comme la ressource fondamentale; la première école «focuse(s) on the development of relationships and the building of understanding between peoples », le pouvoir étant un «venal control ${ }^{30}$. Il s'agit de transformer ce conflit, c'est-à-dire à la fois d'en supprimer la manifestation violente et d'en traiter les causes pour installer une paix juste. Les promoteurs de la paix positive revendiquent par conséquent la visée ambitieuse du changement de la société élargie, à tous les niveaux : en amenant les élites et les gens ordinaires à embrasser d'autres convictions sur la guerre et la paix, ainsi que des récits communs. Les programmes travaillent à toutes les échelles : celles des institutions, du rapport entre citoyens et institutions, des normes collectives, des relations interpersonnelles, mais aussi des vertus individuelles.

Ce sont ces objectifs ambitieux formulés par des universitaires, des experts et des militants (formés dans les universités), contestant le monopole de l'État sur la régulation des conflits, qui ont, depuis la fin de la Guerre froide, fait progressivement leur chemin dans les recommandations de l'ONU déjà évoquées. Les OI et les États affichent au milieu des années 2000 un large programme de peacebuilding, qui intègre sécurité, négociation politique et réforme institutionnelle, et développement socio-économique y compris dans ses aspects «transformateurs »du conflit ${ }^{31}$. La paix positive est en outre devenue un indicateur, opposable à d'autres indicateurs de développement ou de paix : 1'Institute for Economics and Peace classe les pays du monde selon un Global Peace Index, dont se distingue un Positive Peace Index ${ }^{32}$.

Une proposition d'action internationale en faveur de la paix, de nature critique, esquissée au sein des universités à partir des années 1950, est donc parvenue à se faire une place, moins affirmée que celle des actions diplomatiques et militaires mais visible et cohérente, dans le programme d'action des grands acteurs internationaux. Comment établir le lien entre l'émergence de la première et cette généralisation ? L'articulation est d'autant plus malaisée que les études existantes ne portent pas sur l'ensemble des acteurs susceptibles d'être pertinents. Certains inventaires recensent les grandes OI et une poignée d'États, tandis que d'autres mettent l'accent sur les $\mathrm{ONG}^{33}$. Et si des travaux académiques permettent de mieux comprendre le développement de la peace research, les liens et filiations pointés avec des institutions internationales ou des mobilisations transnationales, le sont sur la base - à la

\footnotetext{
${ }^{27}$ D. Sharp rappelle l'égalitarisme vigoureux de Galtung, qui « reflects an almost visceral aversion to dominance, imposition, and "top dogs" and a general skepticism about structure, hierarchy, and organization more generally », «Positive Peace, Paradox, and Contested Liberalisms », art. cité, p. 124.

${ }_{28}$ Eilish Rooney et Fionnuala Ni Aolain, « Transitional Justice From the Margins: Intersections of Identities, Power and Human Rights », International Journal of Transitional Justice, 12(1), 2018, pp. 1-8.

${ }^{29}$ E. Franklin Dukes, «Why Conflict Transformation Matters: Three Cases », Peace and Conflict Studies, 6(1-2), novembre 1999, pp. 1-16.

${ }^{30}$ William I. Zartman, «Conflict Management: The Long and the Short of it », SAIS Review, hiver-printemps 2000, pp. 227229.

${ }^{31}$ M. Barnett, H. Kim, M. O’Donnell et L. Sitea, «Peacebuilding: what is in a name? », art. cité, montrent que la plupart des grands acteurs revendiquent une action de peacebuilding, y compris orientée vers une paix positive : ONU, Union européenne, institutions financières internationales, États-Unis, Royaume-Uni, Allemagne, France, Canada et Japon.

${ }^{32}$ Voir le classement à https://www.economicsandpeace.org/reports/. Consulté le $1^{\mathrm{er}}$ mars 2021.

${ }^{33}$ Voir par exemple M. Barnett et al., «Peacebuilding: What Is in a Name? », art. cité, et les deux réseaux pris pour points d'entrée.
} 
valeur probatoire faible - de la similarité des concepts et de l'évidence supposée de la diffusion des idées ${ }^{34}$.

Deux choix méthodologiques simples ont été faits ici. Nous sommes, d'une part, entrée par les organisations-charnières plutôt que par un acteur supposé dominant - par contraste avec les démarches classiques de relations internationales aussi bien qu'avec les enquêtes de sociologie de l'international ${ }^{35}$. Cet article s'appuie sur une série d'enquêtes portant sur des ONG internationales. Celles-ci n'ont pas été appréhendées comme des acteurs singuliers aux caractéristiques nettement distinctes d'autres acteurs des politiques internationales. D'une part, elles sont un élément de la chaîne d'intervention internationale; elles suggèrent des formes d'action aux OI et États, mettent en œuvre leurs politiques, sont subventionnées par eux, etc. D'autre part, les salariés des ONG semblent avoir moins de caractéristiques distinctives que ce n'était le cas dans les organisations pionnières de l'humanitaire comme Médecins du Monde ou Médecins sans Frontières ${ }^{36}$. Les ONG sont bien souvent un moment dans la carrière des acteurs internationalisés, avant ou après un emploi dans une OI, une administration, une association locale, un cabinet d'avocats, etc. Elles ne sont donc pas ici des objets en soi, mais des mécanismes intermédiaires très diversifiés - en termes de tailles, de rôles, de statuts, mais aussi de positionnement en regard des secteurs que sont les OI/États, l'espace académique, l'univers militant et religieux, ou encore du point de vue des caractéristiques sociologiques de leurs acteurs.

\section{Encadré méthodologique}

Cet article se fonde sur un retour sur les données collectées dans plusieurs recherches portant sur les politiques internationales de sortie de conflit, entamées en 2007. Outre l'analyse documentaire, plus de soixante entretiens ont été menés aux sièges des organisations internationales (inter- et non gouvernementales) concernées, c'est-à-dire à Bruxelles, Genève, Londres et plusieurs villes de la côte Est des États-Unis, ainsi que sur deux de leurs terrains d'intervention, à Bogota en Colombie (en 2009 et 2013) et à Bujumbura au Burundi (dans le cadre d'une enquête collective, entre 2009 et 2013). L'histoire des organisations et les trajectoires d'agents des organisations non gouvernementales ont été reconstituées par voie notamment d'entretiens pour douze de ces ONG (fondateurs et responsables de programmes de Search for Common Ground, Seeds of Peace, Interpeace, Mercy Corps/Conflict Management Group, Conciliation Resources, International Alert, Initiatives of Change, World Vision, Mennonite Central Committee, Collaborative for Development Action, Collaborative Decision Resources et International Center for Transitional Justice). Plusieurs de ces organisations et de leurs relais locaux ont par ailleurs donné lieu à des observations (un stage de formation, des colloques et rassemblements, des activités de transformation des conflits sur les terrains d'intervention $)^{37}$. Les enquêtes, prises ensemble, sont donc des sites d'observation différenciée d'acteurs relativement analogues en termes de positions structurelles, de trajectoires et de propositions, au sein d'une même arène de la transformation des conflits.

L'enquête entendait rendre compte en effet non du fonctionnement d'une organisation particulière ou d'un milieu sur un terrain local donné, mais d'une arène entière, au sens du terme défini plus haut. Le propos n'est pas de délimiter un domaine thématique à partir des concepts et idées maniés par les acteurs. Certes, la quasi-totalité de ces ONG, et de leurs

\footnotetext{
${ }^{34}$ Ch. Tenenbaum, La médiation dans les relations internationales, op. cité. Le modèle est ici celui des théories constructivistes déjà évoquées. Voir aussi Judith Goldstein et Robert O. Keohane, « Ideas and Foreign Policy: An Analytical Framework, dans des mêmes, ed., Ideas and Foreign Policy. Beliefs, Institutions, and Political Change, Ithaca, Cornell University Press, 1993.

35 Y. Dezalay et B. G. Garth, La mondialisation des guerres de palais, op. cit., qui affirment la centralité du champ académique des États-Unis.

${ }^{36}$ Pascal Dauvin et Johanna Siméant, Le travail humanitaire. Les acteurs des ONG, du siège au terrain, Paris, Presses de Sciences Po, 2002.

${ }^{37}$ Voir de l'auteure, « Du droit à la paix », art. cité.
} 
agents en entretiens, font usage des expressions de paix positive et de transformation des conflits. Mais le point d'entrée est ici l'ensemble des liens forgés entre les organisations ou dans le cours des carrières des agents. Les organisations recensées appartiennent, d'une part, aux mêmes réseaux. Mentionnons deux de ces réseaux suffisamment distincts pour consolider la démarche. D'une part, l'Alliance for Peacebuilding (AfP), installée à Washington D.C. et créée en 2003, rassemble 130 membres, dont 98 aux États-Unis. Les membres sont très diversifiés - de la petite association locale à des ONG très reconnues dans l'espace international, du mouvement religieux au think tank expert, de l'organisation humanitaire à l'université. D'autre part, l'European Peacebuilding Liaison Office (EPLO), installé à Bruxelles et créé en 2001, rassemble aujourd'hui 40 membres des États-Unis et surtout de 16 pays d'Europe. La liste retenue ici est constituée des plus visibles des organisations qui appartiennent aux deux réseaux (les organisations étudiées représentent environ un sixième des organisations de $\left.1^{\prime} \mathrm{AfP}\right)^{38}$. Notons que ces réseaux n'incluent pas de très gros acteurs de l'intervention internationale : on n'y compte ni OI ni ONG de défense des droits humains ; World Vision et Caritas sont les seules organisations d'aide au développement. L'inclusion de nombreuses universités par l'AfP atteste par ailleurs les relations fortes entre cette arène et le monde académique.

L'analyse des trajectoires de certains des agents de ces organisations vient ensuite confirmer les interrelations entre elles, qui forment ensemble une arène, c'est-à-dire non un champ structuré (par une autonomie ou par le rapport à un État dominant, par exemple) mais un espace international cohérent - en termes de circulations, de pratiques et de principes d'action - et néanmoins diversifié sur le plan des pays et des secteurs sociaux d'origine ${ }^{39}$.

\section{Ce que font les pacificateurs après la violence}

Formant ensemble une arène, alliées dans des réseaux et parfois dans des programmes financés par des OI et des États, soucieuses toujours de se démarquer par rapport aux modalités négatives et élitaires de pacification, ces organisations ne partagent pas seulement des idées et des concepts. Leurs pratiques convergent aussi. Les acteurs interrogés aux sièges des organisations évoquent dans le temps court d'un entretien des expériences très diverses : médiation de paix en Colombie, négociations politiques en Afrique du Sud, tenue d'un débat public sur la pêche en Nouvelle-Angleterre, gestion d'un conflit foncier au Guatemala, dialogue avec de jeunes délinquants d'un quartier pauvre de Boston ${ }^{40}$, guerre au Kosovo et cellule de crise post-ouragan à la Nouvelle-Orléans ${ }^{41}$, campagne anti-armement dans les villes des États-Unis, médiation auprès des Natives dans le même pays, formation au déminage ou négociations en Israël-Palestine ${ }^{42}$.

S'ils peuvent embrasser dans un même geste autant de conflits et de lieux différents, c'est parce les techniques qu'ils emploient leur semblent partout les mêmes, et qu'elles sont unifiées, d'une part, par l'intention critique sur laquelle on reviendra, et d'autre part, par des doctrines et des figures communes de fondateurs - pour les uns Roger Fisher, « the guru of conflict resolution ${ }^{43}$, pour les autres, Galtung ou même Gandhi ${ }^{44}$. Bien sûr, les programmes

\footnotetext{
${ }^{38}$ Voir https://www.allianceforpeacebuilding.org et http://eplo.org/about-us/member-organisations/\#toggle-id-31.

39 D'autres indices ont permis de confirmer l'appartenance de ces organisations à une même arène, et notamment la consultation d'annuaires spécialisés ou l'analyse de certains programmes d'activités des OI, qui peuvent les associer (voir infra).

${ }^{40}$ Entretien avec Jim Tull, consultant, ancien salarié du Conflict Management Group/Mercy Corps (CMG/MC) arrivé à la résolution des conflits après des études commerciales, Boston, 13 février 2007. Marshall Wallace et Diana Chigas, employés de Collaborative for Development Action (CDA), ont respectivement été formés au marketing et à la littérature française et au droit de la famille; c'est par la médiation (ainsi que des connexions religieuses et familiales) qu'ils sont arrivés à l'intervention pour le développement et la paix (Cambridge, 14 février 2007).

${ }^{41}$ Ruth Allen, salariée du CMG/MC formée à la géologie, New York, 20 février 2007. Seema Tikare, de la même organisation, formée à la politique industrielle, évoque en outre les réfugiés, la violence à l'encontre des femmes au Népal, Boston, 14 février 2007.

${ }^{42}$ Grant Rissler, membre salarié du Mennonite Central Committee, juriste, Washington D.C., 2 mars 2007.

${ }^{43}$ Entretien avec Jim Tull, cité.
} 
s'ajustent aux injonctions des bailleurs, aux agendas des relais locaux et à des contextes locaux très variés ${ }^{45}$; les organisations cherchent aussi à se différencier. Mais les convergences sont nettes. Que font ces pacificateurs ? S'ils pointent la nécessité de corriger les mécanismes qui ont conduit à un conflit ouvert (les inégalités de ressources ou la montée de l'intolérance), ils entreprennent surtout d'installer au sein de la société - et de chaque homme - une capacité d'identifier ces failles et d'y réagir à temps. L'action en vue de la paix consiste donc, d'une part, en une éducation des individus. Elle s'efforce, d'autre part, de relier ces hommes en les faisant dialoguer.

Les différentes applications de la peace research sont de nature éducative. Il s'agit de rééduquer les «violents », par exemple en les formant aux droits humains, et non plus seulement de les désarmer ou de les intéresser à court terme à un accord de paix. Mais les pacificateurs entreprennent d'organiser le plus grand nombre possible de dialogues en petits groupes. L'ONG Search for Common Ground revendique par exemple 3,6 millions de personnes directement touchées par ses programmes en 2018, pour 237000 personnes formées en 2017 et plus de 42 millions de personnes touchées la même année par les activités médiatiques ${ }^{46}$. Le propre de ces rencontres interpersonnelles est en effet de favoriser un « effet de levain »: les interactions personnelles suscitées doivent conduire à une diffusion des convictions nouvelles des bénéficiaires dans leurs environnements locaux - auprès des proches, voisins, commerçants, etc. ${ }^{47}$

Ce processus implique bien sûr la diffusion de connaissances : sur l'histoire comme sur les modes de vie et expériences du groupe ennemi. Mais les dispositifs misent surtout sur le dialogue ému. Les commissions de vérité sont par exemple conçues comme des dispositifs de partage de l'émotion dans des espaces publics ${ }^{48}$. Certaines revendiquent une fonction de «guérison » (healing, terme affiché en très grands caractères lors des auditions de la Truth and Reconciliation commission sud-africaine) - des victimes comme des agents de la violence ${ }^{49}$. Dispositifs de justice transitionnelle comme dialogues en petits groupes doivent renforcer l'empathie des bénéficiaires, et ainsi réformer durablement les rapports à autrui dans l'ensemble de la société. Les stratégies diplomatiques de paix négative sont, elles, accusées de ne pouvoir enfanter que des dispositions pacifiées précaires, dans les esprits d'élites changeantes. Le dépassement des préjugés sur l'autre groupe, les liens nouveaux avec l'ancien ennemi supposé, produiraient une attitude de tolérance bien implantée. Celle-ci ferait obstacle le moment venu à la répétition des violences. Une tel pari, notons-le pour y revenir, implique une conception particulière des causes de la guerre : c'est dans le for intérieur des individus que se tapirait le futur tueur, et que se replierait une victime traumatisée, elle-même suspecte de vengeance. Les pacificateurs s'efforcent donc de combattre les préjugés et les passions idéologiques et racistes, données pour causes des violences collectives. Si la guerre est comprise comme un processus collectif, ce qui l'alimente comme ce qui peut la prévenir semble avoir son siège dans les dispositions individuelles.

Outre ces actions d'éducation empathique, la paix positive s'incarne dans de multiples initiatives pour faire dialoguer les membres des sociétés post-conflit, et par là produire des liens durables, de la confiance et un monde commun. Villageois, écoliers, entrepreneurs, femmes sont réunis pour dialoguer, tandis que victimes et auteurs des violences politiques sont

\footnotetext{
${ }^{44}$ «I learned about Gandhi, I learned about Quakerism... », entretien avec Mary Anderson, CDA, Boston, 19 février 2007. L'appartenance à la dénomination quaker est la clé de la constitution de CDA.

${ }^{45}$ Roger Mac Ginty, International Peacebuilding and Local Resistance: Hybrid Forms of Peace, New York, Palgrave Macmillan, 2011 ; Gearoid Millar, An Ethnographic Approach to Peacebuilding. Understanding Local Experiences in Transitional States, Londres, Routledge, 2014.

${ }^{46}$ Selon ses déclarations à la base de données GuideStar : https://www.guidestar.org/profile/52-1257425.

${ }^{47}$ J. P. Lederach, The Moral Imagination, op. cit.

${ }^{48}$ Y compris par l'ONU, Haut-Commissariat aux droits de l'homme, Outils de l'État de droit pour les sociétés sortant d'un conflit : commissions vérité, New York, Nations Unies, 2006.

${ }^{49}$ De l'auteure, «Pleurer ensemble restaure-t-il le lien social ? Les commissions de vérité, "tribunaux des larmes" de l'aprèsconflit », p. 199-226 dans R. Nollez-Goldbach et J. Saada (ed.), La justice pénale face aux crimes de masse. Approches critiques, Paris, Pedone, 2014.
} 
incités à échanger devant les commissions de vérité. Or le lien social qu'il est question de restaurer ou de créer est défini comme un lien interpersonnel. Dans le dialogue local, en petits groupes, ce ne sont pas les rapports aux institutions qui sont visés, mais les interactions horizontales entre les personnes. C'est ainsi que la justice transitionnelle entend " remodeler les identités collectives ${ }^{50}$, et les dialogues produire «un nouvel ethos encastré dans une culture de paix $»^{51}$.

\section{À quelle violence politique veulent mettre fin les promoteurs de la paix positive ?}

Appréhendée depuis ces pratiques, une paix positive exigerait une rééducation des personnes susceptible de venir à bout des méfiances et des haines, d'une part, un lien fort, dense et transversal, qui ferait naître un accord sur les divisions passées et sur un monde commun, d'autre part. Ce diagnostic d'experts condense ce que beaucoup pensent des violences politiques. Elles apparaissent d'abord et surtout comme destructrices : des hommes et des choses - ce qu'elles sont -, mais aussi des liens, des institutions, et du fond moral des personnes. La guerre serait le contraire de la paix, et celle-ci naîtrait d'un lien social dense au sein des groupes sociaux et entre eux. Or, les sciences sociales disent autre chose. Elles remettent en question cette conception exceptionnaliste et exclusivement destructrice des guerres.

On sait, d'une part, que les violences politiques ne sont pas le produit de haines anciennes et incorporées par les individus. Elles sont au contraire enchâssées dans des interactions sociales ordinaires ainsi que dans l'existant moral, dont elles suscitent la révision sur la base de ce que semble requérir la situation et des prescriptions de l'autorité ${ }^{52}$. C'est même la manière plus générale dont les pacificateurs dépeignent le comportement humain qui peut être discutée. Les violences, mais aussi la paix négative, sont en effet imputées à la constance des dispositions et comportements individuels, de la persévérance de la haine de l'ennemi. Or les sciences sociales montrent que l'homme est inconstant, que les comportements varient selon les mondes sociaux traversés et les situations affrontées ${ }^{53}$. Nous nous comportons moins en extériorisant des dispositions constantes qui définissent notre personnalité, qu'en regardant les autres agir. C'est donc le principe d'une action pédagogique visant la réforme durable des dispositions de l'individu qui peut être questionné ${ }^{54}$.

Les travaux récents ont par ailleurs montré que, dans le feu de la violence, les échanges sociaux ordinaires se prolongent, et avec eux des rapports sociaux hiérarchisés ${ }^{55}$. Ils nous apprennent que des sociétés peuvent fonctionner durablement de façon violente, qu'il n'y a pas de frontière si nette entre guerre et paix, et que la violence déchaînée n'est pas étrangère à la violence routinière et contenue ${ }^{56}$. Bien sûr, les guerres blessent et certaines peuvent priver tout homme de sécurité, lorsque tout « tacite » (c'est-à-dire toute connivence, toute convention implicite), et avec lui toute stabilité, disparaissent ${ }^{57}$. Mais une forme de routinisation de la vie

\footnotetext{
${ }^{50}$ Pierre Hazan, Juger la guerre, juger l'Histoire. Du bon usage des commissions vérité et de la justice internationale, Paris, Presses universitaires de France, 2007, p. 31.

${ }^{51}$ Yaacov Bar-Siman-Tov et Gemma H. Bennink, «The Nature of Reconciliation as an Outcome and as a Process », dans Y. Bar-Siman-Tov, ed., From Conflict Resolution to Reconciliation, Oxford, Oxford University Press, 2004, p. 38.

${ }^{52}$ Harald Welzer, Les exécuteurs : des hommes normaux aux meurtriers de masse, Paris, Gallimard, 2007.

${ }^{53}$ On peut renvoyer, à ce sujet, à Erving Goffman, Les cadres de l'expérience, Paris, Minuit, 1991 (1974) ; Bernard Lahire, L'homme pluriel. Les ressorts de l'action, Paris, Armand Colin, 2001 ; Timothy Tackett, Par la volonté du peuple. Comment les députés de 1789 sont devenus révolutionnaires, Paris, Albin Michel, 1997 ; Paul Veyne, Les Grecs ont-ils cru à leurs mythes? Essai sur l'imagination constituante, Paris, Le Seuil, 1983 ; John M. Doris, Lack of Character: Personality and Moral Behavior, Cambridge, Cambridge University Press, 2008.

${ }^{54}$ Sarah Gensburger et Sandrine Lefranc, À quoi servent les politiques de mémoire ?, Paris, Presses de Sciences Po, 2017.

${ }^{55}$ Nicolas Mariot, «"Je crois qu'ils ne me détestent pas". Écrire l'inimitié dans les correspondances lettrées de la Grande Guerre », Genèses, 96, 2014, pp. 62-85

${ }^{56}$ Marielle Debos, Le métier des armes au Tchad: le gouvernement de l'entre-guerres, Paris, Karthala, 2013 ; Roland Marchal, «Les frontières de la guerre et de la paix », Politix, 58, 2002, pp. 39-59.

${ }^{57}$ Par exemple lors de la guerre de 1976-1992 au Mozambique décrite par Carolyn Nordstrom, « Terror Warfare and the Medicine of Peace », Medical Anthropology Quarterly, 12(1), 1998, pp. 103-121. Les violences y sont si systématiques qu'elles suppriment toute chance de vie ordinaire : «We can't walk freely, we can't work freely, we can't eat freely, we can't
} 
sociale demeure possible, et des formes nouvelles de contrôle collectif et des routines s'organisent, qui permettent l'évitement par la plupart du basculement dans la violence ${ }^{58}$.

Le caractère exceptionnel de la violence politique ne fait guère de doute aux yeux des promoteurs de la paix positive. La violence ouverte est considérée comme l'envers de la normalité ; la guerre s'oppose strictement à la paix. Les sciences sociales qui ont exploré les processus sociaux notamment violents montrent pourtant que les continuités parfois l'emportent sur les discontinuités ${ }^{59}$. Si les conflits violents ne sont pas ces phases de vide, de chaos ou d'anomie que l'on présume, si la guerre peut être «positive», pourquoi faudrait-il voir dans les moments qui suivent, une opportunité de rétablir des liens sociaux de proximité qui sont supposés avoir été emportés, de ré-humaniser les hommes et de reconstruire le lien social?

Certaines enquêtes menées en Colombie confirment la fragilité de telles hypothèses, et par exemple l'idée qu'il est nécessaire d'adopter des politiques qui amènent les individus à surmonter des haines installées par un travail de restauration du dialogue et de la confiance ${ }^{60}$. On présume sans doute trop vite que les ennemis d'hier demeurent les ennemis d'aujourd'hui, qu'ils sont incapables de renouer des liens fonctionnels, sinon affectifs. Une étude sur la coexistence entre anciens combattants démobilisés, déplacés et victimes de guerre dans des quartiers de trois villes colombiennes (Bogotá, Medellín et Valledupar) ${ }^{61}$ met au contraire en évidence la fréquence de leurs interactions sociales, leur sentiment de proximité, l'absence de tout rejet mécanique et l'importance des différences de traitement perçues. Loin d'affirmer que l'harmonie succède à la guerre, l'enquête montre que c'est un lien social ordinaire, quotidien, qui est expérimenté dans ces contextes de sortie de conflit, à bonne distance des prémisses comme des appropriations politiques des programmes de paix positive.

\section{Comment une proposition critique rallie et organise des acteurs internationaux}

\section{Les programmes de transformation des conflits violents ne sont pas le produit d'une intention politique}

Comment expliquer la diffusion d'une telle conception hypostasiée de la violence par des acteurs pourtant formés aux sciences sociales, familiers souvent des études des processus de guerre et de paix? L'un des arguments fréquemment employés est celui de la demande locale. La transformation des conflits répondrait mieux que d'autres modèles aux besoins et cultures des sociétés post-conflit ${ }^{62}$. Cet argument convainc peu, tant les programmes observés, conçus souvent dans un contexte étatsunien, restent portés par des acteurs étrangers. Les organisations sont par exemple soucieuses de former des formateurs locaux, mais font généralement, et longtemps, intervenir à cette fin des experts étrangers ${ }^{63}$.

Il n'est pas non plus possible d'expliquer la généralisation des programmes de transformation des conflits par la force des mobilisations transnationales d'acteurs convaincus ${ }^{64}$. Bien sûr,

live freely », disent des personnes interviewées par la chercheuse, pp. 107-110. Et pourtant, celle-ci insiste sur le fait que les gens « re-create viable worlds ».

${ }_{58}$ C'est ce que vérifie Teresa Koloma Beck dans son ethnographie du conflit angolais : The Normality of Civil War, Frankfurt, Campus Verlag, 2006.

${ }^{59}$ Michel Dobry, Sociologie des crises politiques, op. cit.

${ }^{60}$ Patricia Shields et Joseph Soeters, «Peaceweaving: Jane Addams, Positive Peace, and Public Administration », The American Review of Public Administration, 47 (3), 2015, pp. 323-39 : "Viewing peace as "not violence" may also suggest that peace does not coexist alongside violence in complex, mosaic patterns or that peace-violence is some kind of on-off button », p. 324.

${ }^{61}$ Juan Diego Prieto, «Together after War While the War Goes On: Victims, Ex-Combatants and Communities in Three Colombian Cities », International Journal of Transitional Justice, 6(3), novembre 2012, pp. 525-546.

${ }^{62}$ John P. Lederach, Preparing for Peace: Conflict Transformation Across Cultures, New York, Syracuse University Press, 1996.

${ }^{63}$ Diana Francis décrit son expérience de formatrice dans People, Peace and Power: Conflict Transformation in Action, Londres, Pluto Press, 2002.

${ }^{64}$ M. E. Keck et K. Sikkink, Activists Beyond Borders, op. cit. 
beaucoup de ces acteurs sont des militants, mais cette arène internationale, en se professionnalisant, donne une importance de plus en plus grande aux salariés, enthousiastes sans doute, mais polyvalents et susceptibles de rejoindre ensuite des organisations aux interventions sans rapport avec la paix positive ${ }^{65}$. Les entretiens réalisés une fois le poste dans une organisation de paix quitté sont de ce point de vue révélateurs : les pacificateurs ne sont pas dupes ; ils doutent de l'efficacité de ce qu'ils font. Beaucoup, interrogés sur l'évaluation de l'efficacité de leurs pratiques, mettent ainsi l'accent sur leur nature de pari.

«I would say that impact analysis in peacebuilding is the most difficult and unresolved issue in the peacebuilding world (...) what you're doing is making some contribution to it, but we all have to be humble enough to not claim credit for everything that is going on, and the work that is done by us $» 66$.

«J'ai des questions sur la vérifiabilité de l'impact, la quantification de tout ça. (...) Search [for Common Ground] a le réflexe de se réfugier dans l'émotionnel, les petites histoires. (...) Tout ça est pensé en américain. Au bout du compte, c'est une organisation américaine. Ça sort de cerveaux américains, et c'est dur de le traduire en français, en pensée vieux continent. Ils sont très friands de petites histoires, enfants soldats, etc. mais ça compose un tableau assez pointilliste. Mais comment quantifier l'impact, l'articulation avec d'autres dimensions politiques et économiques ? ${ }^{67}$

Ces acteurs savent que beaucoup de leurs interventions, et notamment les plus anciennes, n'ont pas eu le succès escompté ; le conflit israélo-palestinien est ainsi l'une des cibles privilégiées - et incontestablement un échec partagé - de l'ensemble des organisations évoquées ici. Les statistiques de reprise des conflits sont bien connues d'elles ${ }^{68}$, de même que la faible coordination entre les acteurs de la reconstruction post-conflit. Les pacificateurs sont, enfin, très au fait des problèmes d'instrumentalisation dont les programmes peuvent faire l'objet, captés ici par des responsables politiques encore acquis à une logique de guerre, ou là par une ethnie dominante qui fait recruter les siens ${ }^{69}$. Ils perçoivent en outre les effets des intérêts d'États sur les actions des organisations. L'ancien employé de Search for Common Ground, interrogé avant et après son recrutement par l'agence des Nations Unies qu'il souhaitait depuis longtemps rejoindre, expliquait ainsi que le programme qu'il mettait en œuvre dans un pays du Maghreb servait moins la paix - le pays n'avait pas connu de conflit ouvert - que l'alliance avec les élites locales dans le contexte de l'adoption d'un accord de libre-échange avec les États-Unis ; la promotion du dialogue servait en effet dans ce cas la libéralisation du droit civil au travers de la diffusion de la résolution alternative des litiges. Il indiquait en outre que le programme se distinguait au fond assez peu des autres formes d'action internationale : «C'est quand même un peu du développement, ce qu'on fait ». Il pointait, enfin, le caractère aléatoire, et personnel, des terrains d'intervention :

«J'ai jamais eu de réponse sur le pourquoi de la présence de Search au Maroc. Sinon qu'un individu qui a des envies peut créer son domaine, quelle que soit la bigger picture.

\footnotetext{
${ }^{65}$ Voir l'article de l'auteure avec D. Griveaud, « La justice transitionnelle, un monde-carrefour », art. cité.

${ }^{66}$ Entretien par téléphone avec Bill Lowrey, «director of peacebuilding and reconciliation » de World Vision, à Washington D.C., 26 février 2007. Selon Mary Anderson et Lara Olson, de CDA, Confronting war. Critical lessons for practitioners, Cambridge, CDA, 2003, ces acteurs sont «ambivalent about a focus on effectiveness », 8. Elles citent un participant : «It really is not necessary to assess outcomes. We are called to be faithful, to do good, without regard for outcomes. We do what we do because we must do something. To do nothing would be worse ».

${ }^{67}$ Entretiens avec un ancien responsable de programme à Search for Common Ground (SFCG), Bruxelles, 23 février 2005, et Washington D.C., 4 juin 2006, par téléphone depuis les États-Unis à New York.

${ }^{68}$ Selon Arnim Langer, Graham K. Brown et Hanne Albers, le taux de reprise du conflit varie de 35 à $57 \%$ : «Introduction », dans A. Langer et G. K. Brown, Building Sustainable Peace: Timing and Sequencing of Post-Conflict Reconstruction and Peacebuilding, Oxford, Oxford University Press, 2016, p. 3.

${ }^{69}$ L'une des organisations prise en compte dans cet article, CDA, s'est fait connaître par cette critique des effets des interventions, adossée à une revue des programmes d'environ 200 organisations de peacebuilding, en 2001-2002. Mary B. Anderson, Do No Harm: How Aid Can Support Peace-Or War, Boulder, Lynne Rienner, 1999; la même et L. Olson, Confronting war. op. cit.
} 
Après son départ, on gère! Énergies investies, on reste. On essaie de bricoler un argumentaire. Mais compte tenu des difficultés pour lever des fonds (...) Il y a toujours eu des débats internes (...) [C'est] une organisation familiale. (...) le bébé de Johns Marks. Un gourou. Ça me mettait assez mal à l'aise. Quelqu'un d'intéressant, un sacré caractère, il est assez étonnant. Mais que SFCG soit présenté comme l'association fondée par Johns Marks, qu'il en soit le président à vie, que tout soit exprimé à travers le prisme de la pensée de Johns Marks, surtout pour le Moyen-Orient, ça me paraissait extrêmement pesant pour le programme. Et en général je sentais qu'il y avait un manque de distance flagrant, et beaucoup étaient agacés, en séances de brainstorming. Il fallait tuer le père. Mais je n'ai pas senti de remise en cause. L'idée d'être dans la petite boutique de Johns Marks m'agaçait. » ${ }^{70}$

Le contexte international est par ailleurs souvent mis en avant. Le développement des actions de transformation des conflits est dans cette perspective présenté comme une réponse à la multiplication des États défaillants ou de guerres civiles «insolubles » (intractables) - toutes affirmations discutables $^{71}$. Ce développement semble néanmoins explicable dans les termes d'une analyste réaliste; il peut même apparaître comme l'une des dimensions du projet «impérial »des États-Unis ${ }^{72}$, affirmé notamment par le Department of State en $2016^{73}$. La justice transitionnelle, notamment, est une pax americana, la politique d'un État mu par un intérêt stratégique - du moins jusqu'à la forte réduction par le gouvernement Trump des budgets des institutions concernées. Elle est décriée comme une stratégie diplomatique intéressée, dont la souplesse permettrait d'éviter les poursuites contre les nationaux, et de mettre en sourdine les revendications des propres minorités, colonisés ou opposants des ÉtatsUnis $^{74}$. Les programmes favorisant une paix positive sont de même perçus, on l'a vu, par certains acteurs et universitaires, comme une politique favorisant la mise en place d'une élite liée aux intérêts des États-Unis ${ }^{75}$.

Si les diplomates états-uniens y sont dans l'ensemble restés fermés ${ }^{76}$, l'United States Agency for International Development (USAID) s'est beaucoup investie dans les politiques de peacebuilding (330 projets dans 42 pays en 2017) parmi les plus emblématiques du principe de la paix positive. Dans une optique «people-to-people », sur la base d'une théorie du changement fondée sur l'interaction personnelle ${ }^{77}$, l'agence a en effet déployé, dans les années 2010, des activités comme «les programmes de justice restaurative, le dialogue interethnique, la construction de la paix interreligieuse $(. .$.$) et la réconciliation par le bas { }^{78}$. Nombre des programmes déployés par les ONG en Colombie le sont avec son appui.

\footnotetext{
${ }^{70}$ Entretien avec un ancien responsable de programme à SFCG, cité.

${ }^{71}$ Roland Marchal et Christine Messiant, « De l'avidité des rebelles : l'analyse économique de la guerre civile selon Paul Collier », Critique internationale, vol. 16, no 3, 2002, pp. 58-69. Voir l'introduction de ce dossier.

${ }^{72}$ Susanna Campbell, David Chandler et Meera Sabaratnam, A Liberal Peace? The Problems and Practices of Peacebuilding, Londres, Zed books, 2011.

${ }^{73}$ United States Department of State Transitional Justice Initiative, 2016, Transitional Justice Overview, 16 mai, https://www.state.gov/j/gcj/transitional/257566.htm.

${ }^{74}$ Loramy Gerstbauer, U.S. Foreign Policy and the Politics of Apology, New York, Routledge, 2017.

${ }^{75}$ Zachary D. Kaufman, United States Law and Policy on Transitional Justice: Principles, Politics and Pragmatics, New York, Oxford University Press, 2016; Dyonisis Markakis, US Democracy Promotion in the Middle East: Pursuit of Hegemony, Londres, Routledge, 2015, p. 3. D. Sharp y fait aussi reference : « More darkly, it seems possible that attempting to operationalize the Galtungian concept of positive peace as social justice could operate as a kind of Trojan horse, widening the latitude for intervention in a peacebuilding and development world already rife with charges of neocolonialism », «Positive Peace, Paradox, and Contested Liberalisms », art. cité, p. 126.

${ }^{76}$ Cynthia J. Chataway, «In Practice. Track II Diplomacy : From a Track I Perspective », Negotiation Journal, 14(3), 1998, pp. 269-287. Hormis une ouverture relative au cours des années 2000 (Ch. Tenenbaum, La médiation dans les relations internationales, op. cit.) qui ne nous semble pas généralisée et a été remise en cause par le durcissement des gouvernements Obama et Trump.

${ }^{77}$ Krishna Kumar, Promoting Social Reconciliation in Postconflict Societies. Selected Lessons from USAID's Experience, USAID Program And Operations, Assessment Report n²4, janvier 1999.

${ }^{78}$ USAID, Educative Learning Review Synthesis Report: USAID/CMM's People-To-People Reconciliation Fund, Annual Program Statement, Washington, D.C., mars 2014, p. 316.
} 
Comment expliquer qu'une agence d'État assume une politique peu « réaliste »? Le désaveu récent dont elle a fait l'objet de la part du gouvernement Trump est instructif : c'est précisément du fait des tensions internes aux élites de la politique étrangère, aux partis politiques et à l'État fédéral, et pour affirmer une identité distinctive, que l'agence a maintenu cette perspective centrée sur le dialogue en petits groupes. Ce volontarisme met moins en évidence un intérêt national favorable à la transformation des conflits, que des tensions entre les agences. Ce fut le cas, vingt ans auparavant, avec la création de l'United States Institute of Peace, une institution bipartisane, impartiale, qui a largement contribué au développement des intervention en faveur de la transformation des conflits ${ }^{79}$. La paix positive fonctionne ainsi comme une proposition critique interne de la politique étrangère du gouvernement. Elle demeure secondaire dans les pratiques, mais est une ressource importante de différenciation.

\section{Projeter ses conflits et routines locaux}

La transformation des conflits est donc moins le prolongement discret d'une politique impériale que l'une des manifestations des tensions internes aux bureaucraties du foreign policy establishment. C'est donc dans l'État américain et plus largement dans le contexte social local, que cette proposition critique devenue sens commun international, prend sens. On l'a dit plus haut, la paix positive doit inspirer la transformation de conflits indifférenciés : aussi bien le divorce que la grève, les conflits raciaux ou la guerre entre États. Une perspective de sociologie des professionnels de l'international met ainsi en évidence l'importance de l'écologie locale des arènes internationales ${ }^{80}$. L'International Center for Transitional Justice (ICTJ), think tank de référence pour la justice transitionnelle présent partout dans le monde, est par exemple l'un des acteurs d'un débat, aux États-Unis, sur l'«injustice raciale». L'organisation a conseillé la Commission de vérité établie en 2004 par des acteurs de la société civile à Greensboro (Caroline du Nord), autour de l'assassinat en 1979 de militants des droits civiques par des membres du Ku Klux Klan. Elle a pris nettement parti à la fois contre la politique étrangère états-unienne en Irak et contre la « guerre contre le terrorisme ». Initiatives of Change (I\&C) est pareillement investie dans ce qu'elle appelle le «racial healing » aux États-Unis. Search for Common Ground (SFCG) peut compter sur une branche interne, qui a mobilisé les techniques de transformation des conflits autour de sujets de controverse comme l'avortement.

Ces organisations qui s'externalisent ont souvent une histoire très locale. SFCG est par exemple le produit des mobilisations contre les interventions militaires états-uniennes en Amérique centrale.

«(..) l'organisation est présentée comme internationale, par l'implantation géographique et le recours aux locaux. Mais quand on remonte à ceux qui sont aux manettes, ce sont des Américains. Avec des guéguerres entre Washington et Bruxelles, des petites rancœurs sur les procédures financières, des non-dits qui empoisonnaient l'atmosphère. (...) À Bruxelles ou à Bujumbura, tout le monde se reconnaît pas forcément dans l'histoire contre Reagan. C'est sans doute différent à Washington, mais à la racine, [il y a] des considérations de politique intérieures américaines ${ }^{8}{ }^{81}$

Le principe même de la transformation des conflits est d'ailleurs le produit en droite ligne de la lutte pour la survie de la dénomination mennonite - qui fournit des intellectuels, une université spécialisée, l'Eastern Mennonite University, des missionnaires et un bureau du Mennonite Central Committee placé auprès des Nations Unies. Ce groupe anabaptiste s'est en effet efforcé de réaffirmer son identité pacifiste face à des gouvernements généralisant la

\footnotetext{
${ }^{79}$ Rhoda Miller, Institutionalizing Peace. The Conception of the USIP and its Role in American Political Thought, Mac Farland, Jefferson/Londres, 1994.

${ }^{80}$ Voir de l'auteure avec D. Griveaud, « La justice transitionnelle, un monde-carrefour », art. cité.

${ }^{81}$ Entretien avec un ancien responsable de programme SFCG, cité.
} 
conscription militaire. C'est pour justifier un service civique - dans les hôpitaux, les prisons, les villes ségréguées - que les intellectuels mennonites ont systématisé le principe de la transformation des conflits ${ }^{82}$. La dimension locale est donc d'une importance particulière dans la constitution de l'arène de la paix positive, qui est saturée de références aux débats sociaux et politiques en vigueur aux États-Unis.

Au-delà d'un entre-soi nourri de culture américaine, et en particulier d'un ethos protestant individualiste et préférant les relations interpersonnelles à la médiation institutionnelle, ce sont des routines professionnelles locales qui forgent et facilitent la diffusion du principe de la transformation des conflits. L'accent placé sur le dialogue ému entre deux individus et ses effets transformateurs peut ainsi mieux se comprendre si l'on tient compte de la projection et de l'entremêlement de techniques religieuses et psychologiques de conversion des personnes et de gestion du collectif. Par contraste avec les modalités élitaires de pacification, ou avec la réforme des États, l'arène mobilise des techniques utilisées pour de plus petits conflits, dans des secteurs d'activité sans rapport direct avec la violence politique et les guerres.

I\&C, qui fédère des groupes dans 31 pays sur tous les continents, en est un exemple frappant. Il s'agit d'une organisation visible à l'échelle internationale - dotée d'un statut consultatif spécial auprès du Conseil économique et social de l'ONU et membre de la conférence des ONG internationales du Conseil de l'Europe. Cette normalisation ne diminue en rien, toutefois, la singularité d'une organisation créée par un Américain en Grande-Bretagne, dans les années 1930, sous la forme d'un groupe évangélique (Moral Re-Armament). L'organisation a extrapolé des techniques particulières de récit de soi (à partir de la tenue d'un journal intime) et de dialogue, aux conflits syndicaux et politiques. Elle mêle des formes d'expression empruntées aux anabaptistes, le principe évangélique de la conversion personnelle et certaines pratiques thérapeutiques (appliquées aussi par les groupes $\mathrm{d}^{\prime}$ Alcooliques anonymes) ${ }^{83}$. Les rencontres organisées dans un luxueux ancien hôtel à Caux, en Suisse, en sont une illustration : après avoir évoqué chacun leur propre transformation, les représentants des camps ennemis venus de pays en conflit sont amenés à dialoguer, deux à deux. La transformation des personnes, une à une, est supposée, on l'a vu en Colombie, permettre le changement politique durable.

«Le changement personnel est la clé de la démarche d'I\&C (...). Le conflit se désamorce lorsque chaque individu impliqué se convainc de l'importance de son propre changement et décide de s'ouvrir à l'autre ${ }^{84}$.

Ce qui est frappant ici est le réemploi de techniques d'abord conçues pour la résolution de conflits interpersonnels, et même souvent conçues pour contourner l'État et ses modes de régulation des conflits. C'est un trait commun à l'ensemble des programmes de transformation des conflits, y compris sous leurs formes les plus institutionnalisées. Tous mobilisent des compétences et des techniques expérimentées sur le terrain des conflits familiaux, de quartier, raciaux, du travail. Les commissions de vérité empruntent ainsi davantage au registre psychologique qu'à celui du droit - ce que l'enrôlement de psychologues locaux, accoutumés aux conflits conjugaux plus qu'aux assassinats politiques, explique mieux que l'hypothèse peu convaincante selon laquelle les guerres font leur lit dans nos esprits maladivement affectés par la haine... De nombreux agents internationaux continuent de s'investir dans la transformation de petits conflits domestiques. C'est cette constante réutilisation de techniques psychologiques routinières, familières au grand nombre, par des acteurs engagés sur plusieurs fronts, qui conforte la singularité des modalités d'action observées, et notamment l'importance donnée

\footnotetext{
${ }^{82}$ De l'auteure, « Des pacificateurs inspirés... », art. cité. L'universitaire et expert mennonite Lederach est une référence dans cette arène.

${ }^{83}$ Philip Boobbyer, The Spiritual Vision of Frank Buchman, University Park, Pennsylvania State University Press,2013.

${ }^{84}$ I\&C, Méthodologie de facilitation pour la paix et la bonne gouvernance, août 2013, p. 8. Dick Ruffin, vice-président de l'association, revendique au cours de l'entretien d'avoir vécu pareille transformation personnelle, coûteuse pour sa «old southern family », entretien cité.
} 
aux dispositions individuelles et aux relations interpersonnelles. On s'explique mieux ainsi l'erreur de diagnostic sur la nature de la violence politique - parfois traitée comme le serait des violences conjugales.

Il ne s'agit pas de dire, toutefois, que ces activités en bonne partie conduites par des acteurs expatriés sont le simple reflet, et seulement le reflet, de jeux sociaux locaux ${ }^{85}$. C'est bien la structure de l'arène internationale qui explique la force des résonances locales. La précarité sur les marchés du travail expert international, la vive concurrence entre les organisations pour les financements, mais aussi le fait que les pacificateurs expatriés vivent en «bulle », renforcent ce poids des références locales ${ }^{86}$. Les entretiens ont montré que les agents anticipent une mobilité vers un autre domaine voire un retour dans le pays d'origine; leur passage par les politiques de paix n'a pas nécessairement d'effet de spécialisation ni de maintien dans l'arène internationale. C'est un phénomène plus général : le passage des jeunes diplômés d'un milieu professionnel à un autre (du public au privé, des universités du Nord aux terrains du Sud puis parfois aux problèmes sociaux du Nord, etc.) accélère la fluidité de la circulation de thématiques, de normes et d'aptitudes, mais aussi de clés de compréhension des questions sociales. Les agents des organisations de transformation des conflits peuvent ou non être enthousiastes ; la brièveté de leur passage dans ces organisations les amènent, on l'a vu avec l'extrait d'entretien plus haut, à entériner des principes d'action à l'efficacité et à la pertinence desquels ils peuvent ne pas croire ${ }^{87}$. Les OI et les gouvernements, en s'appropriant ces concepts et techniques, participent de ce processus. Ces dispositifs sont donc le produit aussi de processus qui ont lieu au cours de leur internationalisation.

\section{Jeux internationaux et locaux en miroir}

Les ONG spécialisées sont, on l'a dit, relativement périphériques par rapport aux acteurs dominants du jeu réaliste de l'intervention dans les conflits; les interventions militaires, les négociations d'accords de paix, les réformes institutionnelles ont davantage de visibilité. Elles sont aussi moins centrales et moins dotées que certaines ONG internationales plus généralistes qui interviennent dans les conflits violents. Elles portent, en regard de ces acteurs centraux, une proposition critique toujours renouvelée, où une première génération (opposée au modèle de la gestion des conflits, par exemple) se mêle en s'effaçant un peu à une $2^{\mathrm{e}}$ voire une 3 è générations (inspirées par le genre ou la justice sociale) ${ }^{88}$. Roland Paris, qui est l'un des auteurs critiques du «liberal peacebuilding », en est même venu, à la fin des années 2000, à s'inquiéter de l' «hyper-critique » et à entreprendre de «sauver» le modèle qu'il avait le premier pourfendu ${ }^{89}$. Il ne faudrait pas en déduire pour autant qu'il s'agit d'acteurs marginaux, et a fortiori de parias mis de côté pour leurs positions dissidentes. Ce que portent ces acteurs, c'est à bien des égards un programme complémentaire des interventions humanitaires, militaires et de développement et des paix élitaires.

Ces ONG sont ainsi pour beaucoup bien implantées dans le monde des OI, qui souvent les subventionnent, et profondément ancrées dans la chaîne des interventions internationales. Elles ont toutes un grand nombre de partenaires. La plupart interviennent dans des pays nombreux (plusieurs dizaines pour SFCG, I\&C et l'ICTJ). Des efforts explicites d'unification de l'arène, au travers de la création de plateformes décernant des certifications (comme les réseaux évoqués) ou de l'organisation de conférences (à l'instar du Global Partnership for the Prevention of Armed Conflict en 2005 au siège new-yorkais de l'ONU, avec 900 délégués

\footnotetext{
${ }^{85}$ Yves Dezalay et Bryant G. Garth, ed., 2002, Global Prescriptions. The Production, Exportation, and Importation of a New Legal Orthodoxy, University of Michigan Press, Ann Arbor, p. 307.

${ }^{86} \mathrm{~S}$. Autesserre, Peaceland, op. cit.

87 Anne Le Naëlou, «Parcours de jeunes diplômés dans le champ du développement "miroirs" d'un secteur qui change », Sociologies pratiques, vol. 27, $\mathrm{n}^{\circ} 2,2013$, p. 44. L'analyse prosopographique des carrières le confirme, art. cité de l'auteure avec D. Griveaud, «La justice transitionnelle, un monde-carrefour ».

${ }^{88}$ Voir Michael Newman, Transitional Justice. Contending with the Past, Cambridge, Polity Press, 2019, p. 135 et 150, et Kevin Clements, « Peace Building and Conflict Transformation », Peace and Conflict Studies, 4, 1997, pp. 1-14.

${ }^{89}$ Roland Paris, « Saving Liberal Peacebuilding », Review of International Studies, 30(2), 2010, pp. 337-365.
} 
venus de 118 pays). C'est ce que confirme la restitution synthétique des partenariats entre les ONG concernées et deux acteurs internationaux clés - l'un gouvernemental, les États-Unis (USAID, principalement), l'autre intergouvernemental, l'ONU (le PNUD, principalement).

Tableau. Aperçu du financement des ONG de transformation des conflits par les États-Unis et l'ONU (montants arrondis, en millions de dollars E.-U.)

\begin{tabular}{|c|c|c|c|c|c|c|c|c|c|c|c|c|}
\hline $\begin{array}{l}\text { Organi } \\
\text { sation }\end{array}$ & $\begin{array}{c}\text { Se } \\
\text { ed } \\
\mathrm{s} \\
\text { of } \\
\mathrm{Pe} \\
\mathrm{ac} \\
\mathrm{e}\end{array}$ & $\begin{array}{l}\text { SF } \\
\text { CG }\end{array}$ & $\begin{array}{l}\mathrm{I} \\
\& \\
\mathrm{C}\end{array}$ & $\begin{array}{c}\text { Organi } \\
\text { sations } \\
\text { menno } \\
\text { nites }\end{array}$ & $\begin{array}{c}\text { Concil } \\
\text { iation } \\
\text { Resou } \\
\text { rces }\end{array}$ & $\begin{array}{c}\text { Collab } \\
\text { orative } \\
\text { Decisi } \\
\text { on } \\
\text { Resour } \\
\text { ces }\end{array}$ & $\begin{array}{l}\text { Inter } \\
\text { peace }\end{array}$ & $\begin{array}{l}\mathrm{Me} \\
\text { rcy } \\
\mathrm{Co} \\
\mathrm{rps}\end{array}$ & $\begin{array}{l}\text { Wo } \\
\text { rld } \\
\text { Vis } \\
\text { ion }\end{array}$ & $\begin{array}{l}\text { IC } \\
\text { TJ }\end{array}$ & $\begin{array}{c}\text { Carit } \\
\text { as }\end{array}$ & $\begin{array}{c}\text { Interna } \\
\text { tional } \\
\text { Alert }\end{array}$ \\
\hline $\begin{array}{l}\text { USAID } \\
\text { et État } \\
\text { fédéral } \\
(2008- \\
21)\end{array}$ & $<1$ & 30 & $\begin{array}{l}< \\
1\end{array}$ & $>1$ & $<1$ & $\begin{array}{l}\text { Oui, } \\
\text { NP }^{90}\end{array}$ & 5 & $\begin{array}{l}18 \\
00\end{array}$ & $\begin{array}{l}14 \\
00\end{array}$ & $\begin{array}{l}\mathrm{O} \\
\text { ui, } \\
\mathrm{N} \\
\mathrm{P}\end{array}$ & $\begin{array}{c}2 \\
\text { (hors } \\
\text { projet } \\
\mathrm{s} \\
\text { natio } \\
\text { naux) }\end{array}$ & 12 \\
\hline $\begin{array}{c}\text { ONU, } \\
\text { Peaceb } \\
\text { uilding } \\
\text { Fund } \\
\text { ONU, } \\
2006- \\
2020\end{array}$ & 0 & $>1$ & 0 & 0 & $\begin{array}{c}1,25 \\
\text { avec } \\
\text { War } \\
\text { Child }\end{array}$ & 1,2 & 3,8 & 5,6 & 1,2 & $<1$ & $<1$ & 1,5 \\
\hline
\end{tabular}

Ce tableau, nourri par les rapports et déclarations financières des ONG et par les bases de données des deux organisations ${ }^{91}$, montre que la quasi-totalité des premières ont des partenariats avec les secondes, souvent bien établis. Ces alliances sont dans certains cas très étendues. SFCG, par exemple, affiche 780 agents en 2018 et des partenariats en cours avec 69 fondations et ONG, 15 OI et 19 gouvernements. Même les organisations qui ne bénéficient pas ou plus de subventions, ou n'ont pas de partenariats avec ces agences, sont bien intégrées à l'arène institutionnelle. Seeds of Peace (SoP) et I\&C se financent par l'organisation d'événements (comme des galas), les dons privés ou l'autofinancement par les membres. Mais les responsables de SoP furent un temps proche des gouvernements fédéraux démocrates des États-Unis. Et I\&C international, a, on l'a dit, des connexions importantes avec les OI. Nombre des membres de sa direction et de son conseil sont issus de grandes agences internationales (comme la Croix-Rouge et le Secrétariat général de l'ONU). Enfin, ses 32 antennes nationales peuvent être subventionnées par des gouvernements comme par des OI.

Certaines de ces ONG sont même devenues de véritables portes tournantes de l'arène internationale, au sens où elles forment les personnels et rédigent les rapports des gouvernements et des OI. Leurs agents peuvent se faire recruter par ces derniers. Les partenariats noués permettent aux OI de maintenir leur cap et identité propres dans un contexte de concurrence et de manque de coordination. De même que USAID affirme sa distinction au sein de l'État fédéral des États-Unis, le PNUD, soucieux d'affirmer son identité de spécialiste de la paix et d'acteur de terrain (puisqu'il représente l'ONU dans la quasi-totalité des pays), a

\footnotetext{
${ }^{90}$ Voir par exemple : https://www.land-links.org/wpcontent/uploads/2017/05/USAID_Land_Tenure_LGSA_Inception_Plan.pdf, consulté le $1^{\text {er }}$ mars 2021.

${ }^{91}$ Pour USAID, voir https://data.usaid.gov et https://www.usaspending.gov/recipient. Pour le PNUD, https://www.undp.org/ et http://mptf.undp.org
} 
soutenu un bon nombre des ONG de transformation des conflits ${ }^{92}$, et ainsi affirmé une singularité au cours de conflits entre agences de l'ONU (avec le Department of Political Affairs particulièrement) décrits comme «saignants » par un interviewé. Le peacebuilding fonctionne donc comme un label flou qui permet à chaque agence de valoriser son action ${ }^{93}$. Mais s'il est dans l'intérêt de tous de ne pas discuter précisément les propositions, cette convergence et ces concurrences favorisent la diffusion large de techniques de régulation interpersonnelle et délibérative du conflit politique. Ces circulations, mais aussi la concurrence pour les emplois et subsides, favorise par ailleurs l'intégration des ONG. I\&C, historiquement conservatrice, a ainsi fait alliance avec une organisation polairement opposée : Interpeace, organisation suisse créée par un ancien «gauchiste » (selon son terme) passé par une agence onusienne ${ }^{94}$. Autre illustration, celle de l'évolution de certaines ONG généralistes : Mercy Corps a absorbé le Conflict Management Group ${ }^{95}$, tandis que World Vision et Caritas ont fait leur le principe de la transformation des conflits.

Cette intégration est un processus qui se déploie dans l'arène internationale, mais à la charnière de plusieurs mondes. Elle est en particulier le résultat du double positionnement des experts internationaux qui agissent parallèlement dans l'arène internationale et dans les champs académiques. On sait l'importance du bon usage du savoir critique pour la totalité des professionnels de l'international, et la forte interpénétration des expertises internationale et universitaire $^{96}$. Cette articulation présente toutefois des caractéristiques singulières pour l'arène de la transformation des conflits. Elle concourt à son intégration. La catholique Caritas s'appuie ainsi beaucoup sur les experts universitaires mennonites. I\&C, issue d'un groupe évangélique, associe dans sa formation des universitaires de l'Eastern Mennonite University et d'autres universités qui ont participé du développement de la peace research, comme la School for Conflict Analysis and Resolution de l'Université George Mason. Le monde académique est dans ces cas un espace de convergence.

Ce fonctionnement en miroir sert toutefois des concurrences ${ }^{97}$. Le développement de la transformation des conflits a ainsi été favorisé par la reconversion d'anciens diplomates marginalisés au sein des appareils d'État ${ }^{98}$, qui en sont venus à tenir un discours très critique sur leur corps d'origine ${ }^{99}$. Les pionniers de la paix positive dans le monde universitaire sont eux aussi, on l'a dit, de relatifs marginaux ${ }^{100}$ : ils n'appartiennent pas aux relations internationales, ni au droit ou à la science politique, mais à une entre-discipline fréquentée par des sociologues, des anthropologues, des mathématiciens et beaucoup de psychologues sociaux, souvent animés d'un idéal pacifiste et de convictions religieuses. Il s'agit à l'origine d'une contre-mobilisation, « an essentially intuitive revolt against the "IR Establishment" » ${ }^{101}$. Les universités les plus impliquées dans le peacebuilding sont aujourd'hui encore assez mal placées dans la hiérarchie académique états-unienne. L'exemple du réseau de l'Alliance for Peacebuilding peut à nouveau être mobilisé. Si Harvard y figure, c'est au titre d'une tradition

\footnotetext{
${ }^{92}$ Dennis Dijkzeul, «The UNDP: the Development of Peace ? », International Peacekeeping, 92-119.

${ }^{93}$ M. Barnett, H. Kim, M. O’Donnell et L. Sitea, « Peacebuilding: What is in a Name? », art. cité, p. 53.

94 Entretiens avec Randy et Dick Ruffin, Washington D.C., 27 février 2007 ; avec Matthias Stiefel, War-Torn Societies Program devenu Interpeace, Genève, 17 juin 2005.

${ }^{95}$ ONG issu d'un programme de Harvard. Robert Ricigliano, « Networks of Effective Action: Implementing an Integrated Approach to Peacebuilding », Security Dialogue, 34(4), décembre 2003, pp. 445-462.

${ }^{96}$ P. Dauvin et J. Siméant, Le travail humanitaire, op. cit., et de l'auteure avec D. Griveaud, « La justice transitionnelle, un monde-carrefour », art. cité.

${ }^{97}$ Y. Dezalay et B. G. Garth, La mondialisation des guerres de palais, op. cité.

${ }^{98}$ John Burton était un diplomate australien écarté après une accusation de communisme. Joseph V. Montville a fait une brève carrière dans la diplomatie des États-Unis qu'il désinvestit pour se rapprocher d'I\&C; John McDonald a été le directeur adjoint de l'Organisation internationale du travail, puis affecté à la formation des diplomates étatsuniens, avant de se rapprocher de l'université et de fonder en 1982 une ONG spécialisée.

${ }^{99}$ John W. McDonald, «The Track Not Taken? Personal Reflections on State Department Intransigence and Conflict Resolution », Harvard International Review, 22(3), 2000, p. 68.

${ }^{100}$ Ch. Tenenbaum le montre dans La médiation dans les relations internationales, op. cité

${ }^{101}$ Elise Boulding, « Peace Research: Dialectics and Development », Journal of Conflict Resolution, 16(4), 1972, p. 469.
} 
bien distincte, celle du «win win » et du conflict management de Roger Fisher ${ }^{102}$; le rang moyen des quinze universités adhérentes est le $54^{\text {ème }}$ du 2021 Best National University Rankings (U.S. News).

La transformation des conflits a donc été, et demeure, rejetée par la majorité des diplomates en même temps que peu intégrée aux disciplines les mieux institutionnalisées dans les universités. C'est en tant que telle qu'elle s'est fait une place dans le monde des relations internationales. Les propositions critiques qu'elle a formulées n'ont pas été normalisées et complètement intégrées au jeu ordinaire des relations internationales, ni «nationalisées » comme ont pu l'être les droits humains intégrés à la politique étrangère des États-Unis ${ }^{103}$. Elles demeurent des propositions critiques qui, si elles ne subvertissent pas les pratiques, sont valorisées parce que critiques. Les pacificateurs - au gré de scissions et de récusations des plus «managériaux »d'entre eux ${ }^{104}$ - «radicalisent» leur conception de la paix positive, pour mieux affirmer leur distance à l'égard d'experts réalistes. Ce sont ces affrontements au sein de l'espace académique qui peuvent se répercuter dans les OI et les gouvernements - par exemple lorsque USAID se fait le pionnier d'une des versions les plus centrées sur les interactions interpersonnelles du peacebuilding, au moment où le gouvernement s'engage davantage dans la «guerre contre le terrorisme ». Ces jeux de positionnement en périphérie confortent la visibilité de cette proposition critique qu'est la paix positive, aussi bien que la singularité, et le caractère chimérique, de sa description de la violence et de la paix.

L'arène de la transformation des conflits ne s'est pas développée en conséquence d'un projet «impérialiste» ou d'un contre-projet militant. Les acteurs de la transformation des conflits - formés aux sciences sociales - ne sont pas dupes des limites des programmes qu'ils mettent en œuvre dans des pays hier affectés par des massacres, des guerres civiles de grande ampleur, des génocides. S'ils prétendent œuvrer, dans ces pays, à la diffusion de dispositions individuelles de tolérance ainsi qu'à la création de liens forts entre les groupes hier antagonistes, ce n'est pas qu'ils ignorent la très grande ambition d'un programme de paix positive qui ne trouve pas à s'accomplir dans les pays « en paix » dont ils sont originaires. Ils sont d'ailleurs plus critiques que d'autres des inégalités sociales et raciales et des conflits qui y sévissent. Ils critiquent aussi les politiques qui y sont menées par les États, dans leurs pays comme dans les pays post-conflit. C'est précisément cette critique académique et politique qu'ils exportent, et avec elle une partie des techniques utilisées dans leurs pays d'origine pour «transformer» les conflits - tous les conflits : interpersonnels, sociaux, politiques. Ces propositions critiques, projetées des États-Unis et d'Europe vers les pays post-conflit, valent moins pour les paix qu'elles consolident, que pour leur capacité à rallier des hommes et femmes engagés sur plusieurs fronts, et qui ainsi se renforcent réciproquement et parviennent, parfois, à contenir les politiques négatives et coercitives. Les chimères ne sont pas dépourvues d'efficacité.

\footnotetext{
${ }^{102}$ Roger Fisher et William Ury, Getting to Yes. Negotiating Agreement without Giving in, New York, Penguin Books, 2011 (1981).

${ }^{103}$ Nicolas Guilhot, «Entre juridisme et constructivisme : les droits de l'homme dans la politique étrangère américaine », Critique internationale, 38(1), 2008, pp. 113-135.

${ }^{104}$ Mentionnons par exemple l'éloignement d'une branche scandinave d'inspiration marxiste, à la fin des années 1960, ou le tournant gestionnaire de R. Fisher, juste évoqué. Voir D. Sharp, «Positive Peace, Paradox, and Contested Liberalisms », art. cité, pp. 128-131.
} 